\title{
Correction: The Relationship between Cash Flows and Investment according to Economic Status
}

\author{
[J. Korean Phys. Soc. 76, 109 (2020)] \\ DOI: $10.3938 / j k p s .76 .109$ \\ Jie DENG \\ Division of Business Administration, College of Business, Chosun University, Gwangju 501-759, Korea \\ A-young PARK \\ Division of Business Administration, College of Business, \\ Chosun University, Gwangju 501-759, Korea and \\ Department of Anesthesiology, University of Michigan Medical School, Ann Arbor, MI 48109, USA \\ Gabjin $\mathrm{OH}^{*}$ \\ Division of Business Administration, College of Business, \\ Chosun University, Gwangju 501-759, Korea and \\ Administration, University of Wisconsin-Milwaukee, Milwaukee, WI 53211, USA
}

DOI: $10.3938 / \mathrm{jkps} .76 .356$

In Ref. 12, the author's name should be changed from "P. Petr" to "P. Polak". It should read

[12] P. Polak, R. Sirpal and M. Hamdan, Eur. J. Sci. Res. 86, 319 (2012).

*E-mail: phecogjoh@chosun.ac.kr 